\title{
Die beschleunigte Demokratie - Überlegungen zur Weltwirtschaftskrise
}

Im Fortgang der Moderne bröckeln erstaunlicherweise jene Fundamente, auf deren Basis rationale Entscheidungen getroffen werden können. Den Herausforderungen durch komplexer und dynamischer werdende Steuerungsobjekte steht nationalstaatliche Politik daher zunehmend hilflos gegenüber. Das Ensemble demokratischer Institutionen erscheint insbesondere aus zeitstrukturellen Gründen immer weniger geeignet, soziale Entwicklungen zu regulieren oder zu initiieren. Die Erwartungen an die Politik werden daher in der Gegenwart systematisch enttäuscht. Zur Plausibilisierung unserer These werfen wir in der Folge einen genaueren Blick auf das wirtschaftspolitische Entscheidungsverhalten im Rahmen der Weltwirtschaftskrise 2008/2009.

\section{Politik und Rationalität}

Im Kontext der aktuellen Ereignisse taucht immer wieder die Frage auf, warum sich der Staat überhaupt aus dem Marktgeschehen zurückgezogen hat und nun, in der Krise, seine „Rückkehr“ koordinieren muss. Eine beliebte Erklärung für das Verschwinden der Politik besteht darin, sie als Opfer einer neoliberalen Ideologie zu beschreiben. Danach hat sich der Staat unter dem Druck der Ökonomie ins Exil begeben und von dort aus ohnmächtig auf die heilenden Hände eines freien Marktes gehofft. Diese These ist unseres Erachtens nicht ganz falsch, verkennt aber mindestens zweierlei: Zum einen ist der Staat ja zu keiner Zeit wirklich verschwunden. Er hat vielmehr lediglich seine Gestalt verändert und bemüht sich um einen andersartigen Zugriff auf Ökonomie und Subjekt (Lessenich 2008). Zum anderen ist der zu beobachtende Gestaltwandel politischer Machttechniken durchaus freiwillig zustande gekommen. Die Entfesselung der Finanzmärkte ebenso wie letztlich auch die „Globalisierung“ sind eine gewollte Konsequenz politischer Entscheidungen (Mayntz/Scharpf 2005). Angesichts dieser Tatsache ist das Bild eines ohnmächtigen Staates unterkomplex und daher irreführend. Unserer Auffassung nach sind die Deregulierungen und Privatisierungen der Vergangenheit nur dann zu verstehen, wenn sie (auch) als politische Ausweichmanöver begriffen werden, um der Verantwortung für transintentionale oder gar irrationale Entscheidungen zu entgehen. Aus der hier vorgeschlagenen Perspektive erklärt sich der „Rückzug“ des
Staates aus den Finanzmärkten nicht in erster Linie durch sein großes Vertrauen in die Selbstorganisationskräfte des Marktes oder durch seine Machtlosigkeit gegenüber wirtschaftlichen Interessengruppen, sondern durch die berechtigte Furcht, die Qualität oder Rationalität der eigenen Entscheidungen auf diesem Terrain nicht länger gewährleisten zu können. In Öffentlichkeit und Fachliteratur wird Politik häufig als Feld parteipolitischer Machtkämpfe beschrieben. Nach diesem „realistischen" Politikverständnis könnte man dann etwa derartige Staatsinterventionen (Konjunkturpakete, Teilverstaatlichungen) auf die seinerzeit anstehende, jetzt bereits stattgefundene Bundestagswahl im September 2009 zurückführen. Die hier vertretene Perspektive hebt sich von derartigen Interpretationen $\mathrm{ab}$ und unterstellt „idealistisch“, dass politische Entscheider solche Lösungen anstreben, die das Gemeinwohl fördern. Hierbei sehen sich die Verantwortlichen jedoch zunehmend in eine Situation versetzt, in der die Entscheidungsbasis brüchig und Politik daher zum Glücksspiel wird.

Unser Alltagsverständnis von „Rationalität" lässt sich analytisch in eine prozedurale und eine substanzielle Komponente zerlegen. Substanzielle Rationalität bezieht sich dann auf den erzielten Effekt einer Entscheidung. Hierbei vergleicht man den tatsächlichen Output mit der ursprünglichen Absicht des Akteurs. Sobald das produzierte Ergebnis signifikant von dem originär angestrebten Zustand abweicht, ist man dazu geneigt, der getroffenen Entscheidung nachträglich das Attribut ,rational“ abzuerkennen. Prozedurale Rationalität bezieht sich demgegenüber auf den Entscheidungsprozess, welcher dem ei- gentlichen Entschluss vorausgeht. Dafür ist es wichtig, dass in Bezug auf das Entscheidungsproblem eine Präferenzordnung vorliegt, oder, wie in den meisten Fällen, eine solche erst mühsam hergestellt wird. Hierzu ist eine - oftmals zeitaufwendige - Auseinandersetzung mit den vorhandenen Handlungsalternativen unbedingt notwendig; die Kontingenz der Situation ist zu reflektieren. Je stärker die Komplexität des Entscheidungsproblems im Vorfeld des Entschlusses ausgeblendet wird, der Reflexionsprozess also vorzeitig abgekürzt wird, umso weniger plausibel ist es, von einer rationalen Entscheidung zu sprechen. ${ }^{1}$

Politische Rationalität ist folglich genau dann erreicht, wenn der kollektiven Ent-

\footnotetext{
In den Sozialwissenschaften wird "Rationalität" häufig auf einen der gerade beschriebenen Pole reduziert. Ansätze, die der utilitaristisch-konsequenzialistischen Tradition entstammen (z. B. RationalChoice-Theorien), fokussieren einseitig auf substanzielle Ergebnisrationalität, während Autoren, die das Kantische Erbe bewahren (z. B. Jürgen $\mathrm{Ha}$ bermas oder John Rawls), ihren Blick ausschließlich auf die Rationalität des vorangegangenen Prozesses richten. Unseres Erachtens liegt in beiden Fällen eine Verkürzung vor, die dem alltagssprachlichen Reichtum des Rationalitätsbegriffs nicht gerecht wird.
}

Henning Laux, M. A., wissenschaftlicher Mitarbeiter an der Friedrich-Schiller-Universität (FSU) Jena. Arbeitsschwerpunkte: Gesellschaftstheorie, Politische Soziologie, Entscheidungs- und Netzwerktheorie. e-mail: Henning.Laux@uni-jena.de Hartmut Rosa, Prof. Dr., Lehrstuhl für Allgemeine und Theoretische Soziologie, FSU Jena. Arbeitsschwerpunkte: Zeitsoziologie, Soziologie der Moderne, Kommunitarismus. e-mail: Hartmut.Rosa@uni-jena.de 
scheidung erstens ein sorgfältiger Analyseprozess der situativen Handlungsalternativen vorangegangen ist und wenn zweitens in ihrer Konsequenz ein Zustand erreicht wird, der - gemessen an der kollektiven Präferenzordnung (etwa: lieber weniger Arbeitslose als mehr; Haushaltsüberschuss ist besser als ein Defizit etc.) - zufriedenstellend ist. Da die exakten Umstände und Effekte einer politischen Entscheidung niemals vollkommen durch die Verantwortlichen kontrolliert werden, ist eine unmittelbare Nutzenmaximierung im Feld des Politischen eher unwahrscheinlich. Es erscheint daher sinnvoll, von unterschiedlichen Rationalitätsniveaus, sogenannten „Satisficing“-Levels, auszugehen. Entscheidungen müssen nach dieser Lesart nicht die bestmöglichen sein, um als „rational“ zu gelten: Es genügt, wenn beispielsweise $80 \%, 70 \%$ oder $60 \%$ der prozeduralen und substanziellen Qualitätsansprüche erfüllt werden. Politische Entscheidungen, welche gegen die idealtypischen Kriterien der Akteure verstoßen, sind dann nicht automatisch irrational. ${ }^{2}$ Es handelt sich vielmehr in der Regel um Fälle von „bounded rationality". 3

Im Rahmen dieses Beitrags wird sich zeigen, dass selbst die reduzierten Erwartungen der bounded rationality im Fall nationalstaatlicher Politik strukturell unterboten und enttäuscht werden. In der Folge werden zwei zentrale Ursachen für das $\mathrm{Ab}$ sinken des politischen Rationalitätsniveaus genauer herausgearbeitet: Komplexität und Beschleunigung.

\section{2 \\ Politik und Komplexität}

Es ist kaum bestreitbar, dass Entscheidungen von gesamtgesellschaftlicher Tragweite ein hohes Quantum an Komplexität implizieren. Man denke etwa an Themen wie die Ausgestaltung des Steuersystems, Sterbehilfe, Energiepolitik, entfesselte Märkte, internationale Konflikte oder den Umgang mit Gentechnik. In diesem Sinne stellt sich für die politische Klasse das grundsätzliche Problem spätmoderner Entscheidungsunsicherheit fraglos in radikalisierter Form. Einige der gegenwartsspezifischen Schwierigkeiten möchten wir nun in komprimierter Form darlegen.

\subsection{FEHLENDE GRUNDSATZDEBATTEN}

In einer Studie von Werner Patzelt aus dem Jahre 1994 geben 74 \% der deutschen Bundestagsabgeordneten an, dass sie sich im politischen Tagesgeschäft mehr Platz für Grundsatzfragen wünschen (Patzelt 1996). Wie stabil und erstrebenswert kann eine Wirtschaft sein, wenn sie jedes Jahr wachsen muss? Unterstützt oder untergräbt eine Institution wie die Börse das menschliche Zusammenleben? Inwiefern löst der Markt die mit ihm verbundenen Versprechen ein? Worin bestehen oder bestanden diese Versprechen überhaupt? Welche Maßstäbe von Lebensqualität taugen zur Orientierung der Policy-Entwicklung? Solche Fragen erscheinen idealistisch, naiv, ja sogar grotesk, eine Diskussion oder gar eine Verständigung darüber ausgeschlossen. Und doch ist eine diesbezügliche Verständigung unerlässlich, solange an dem politischen Ziel festgehalten wird, die Welt nach unseren Vorstellungen zu gestalten. Die Idee der rationalen Gestaltung geistert derzeit gerade wieder durch Talkshows, Zeitungsartikel, Aufsichtsräte, Wohnzimmer und Kneipen. Der Staat soll zurückkehren, die Krise beheben und die Weichen für ein besseres Wirtschaftssystem stellen. Im Bezug auf den politischen Entscheidungsprozess bleibt allerdings bereits die Frage nach den anzustrebenden Zielen ein ungelöstes Rätsel.

\subsection{FEHLENDES FACHWISSEN}

Der soeben angedeutete Verlust einer wertbasierten Präferenzordnung wird durch eine massive Krise der sachlichen Wissensbasis flankiert. Es ist kein Geheimnis, dass die politische Agenda eine immer höhere Fachkompetenz von den Volksvertretern verlangt. Diese Entwicklung zeigt sich anhand der weiter wachsenden Zahl an Expertengremien oder dem Siegeszug der Politikberatung im Dunstkreis der Parlamente. Zugleich erfolgt eine nie dagewesene thematische Spezialisierung der einzelnen Parlamentarier. Hinter Phänomenen wie der Fraktionsdisziplin verbirgt sich längst mehr als ein strategisches Machtkalkül: Halbblinde Gefolgschaft ist für Abgeordnete zur letzten Möglichkeit geworden, am Entscheidungsprozess in einer Weise teilzunehmen, die ihnen zumindest die Aufrechterhaltung einer „Rationalitätsfiktion" $"$ erlaubt. Verordnungen und Gesetzestexte sind daher der Sache nach immer häufiger das Produkt einiger weniger Spezialisten in den Parteien und Ministerien. Im Fall der Finanzkrise scheint das besonders deutlich. So wusste im August 2008 vermutlich nur ein Bruchteil der Parlamentarier (und Bürger), was ein Leerverkauf überhaupt ist. Es ist daher nicht überraschend, dass sich zur politischen Bearbeitung der Finanzkrise in Deutschland nur ein äußerst kleiner Zirkel in Berlin zusammengefunden hat. Einer Einschätzung der Wochenzeitschrift Die ZEIT vom 4.12. 2008 zufolge handelt es sich im Grunde sogar nur um drei Personen: Jörg Asmussen (Finanzministerium), Axel Weber (Präsident der Bundesbank) und Jens Weidmann (Kanzleramt). Alle anderen scheinen, zumindest beim Ausbruch der Krise, nur mit großen Abstrichen in der Lage zu sein, die komplexen Vorgänge auf dem internationalen Finanzparkett zu überschauen (Brost et al. 2008). Das größer werdende Nichtwissen schürt das subjektive Gefühl, die Dinge nicht länger unter Kontrolle zu haben. Politische Entschlüsse erhalten nach internalistischen und externalistischen Maßstäben den Geschmack der Beliebigkeit und Willkür.

Die erheblichen Defizite bezüglich der sachlichen Informationsbasis sind jedoch nur ein Aspekt der Wissenskrise in den Parlamenten. Als mindestens ebenso problematisch für das Bestreben nach rationalen Entscheidungen erweist sich die zunehmende Erwartungsunsicherheit bezüglich der Effekte politischer Eingriffe. Die deutsche Bundesregierung hat in Reaktion auf die Weltfinanzkrise ein milliardenschweres Konjunkturpaket aufgelegt, ohne dass irgendjemand zum damaligen Zeitpunkt sagen konnte, ob es einen nennenswerten Ef-

2 Als Umschlagpunkt zwischen rationaler und irrationaler Politik gilt ein Ergebnis, das gerade noch besser ist als jener Zustand, der ohne politische Einmischung erreichbar gewesen wäre.

3 Der Begriff des "Satisficing" geht zurück auf den Wirtschaftswissenschaftler Herbert A. Simon (1946). In seinem Werk wird dieser als Gegenbegriff zur perfekt rationalen Maximierung des Nutzens eingeführt und mit der Idee einer "bounded rationality“ (siehe auch: Simon 1993) verknüpft.

4 Der Begriff wurde in jüngster Zeit von Uwe Schimank (2006) aufgegriffen und wissenssoziologisch präzisiert. Wir verwenden ihn an dieser Stelle jedoch in einem eher alltagssprachlichen Sinne und adressieren damit eine wirkmächtige Selbst- oder Fremdtäuschung von Akteuren, die darin besteht, dass sie kontrafaktisch einen optimal-rationalen Entscheidungsprozess memorieren oder suggerieren. 
fekt auf Konjunktur oder Börsenkurs haben wird. Selbst ex post wird vermutlich jegliche Erfolgs- oder Misserfolgszurechnung reinste Spekulation bleiben. Die Verlängerung der Interdependenzketten im Zuge der Globalisierung lässt allenfalls noch vage Vermutungen über die Auswirkung solcher Entscheidungen zu. Die „ZweckMittel-Reihen " (Simmel) verlängern sich und werden schließlich so unübersichtlich, dass ungewollte Politikergebnisse zum Regelfall werden. ${ }^{5}$ Ökonomische Netzwerke globalisieren sich, transzendieren alte Grenzziehungen und verketten vormals unverbundene Akteure miteinander. Internet und Massenmedien ermöglichen und beschleunigen den weltweiten Kommunikationsfluss.

In „normalen“ Zeiten wie auch in der Krise erweist sich schließlich eine weitere Eigenschaft der spätmodernen Wissensarchitektur als folgenreich: In dem Maße, in dem das Wissen nämlich komplexer wird, altert, sich pluralisiert und heterogene Expertenmeinungen hervorruft, setzt ein Reflexionsprozess ein, der vormalige Gewissheiten untergräbt. Politiker aller Parteien haben aus der Vergangenheit gelernt, dass die Mechanismen der Weltwirtschaft nicht auf simplen Kausalitäten beruhen. Der Rückgriff auf ökonomische Tatsachen ist versperrt. Lehrsätze, wie jene, dass eine Stärkung der Angebotsseite quasiautomatisch zu Arbeitsplätzen und Wirtschaftswachstum führt - oder umgekehrt, dass sich mit antizyklischer Konjunkturpolitik Wirtschaftskrisen dauerhaft vermeiden lassen -, werden als bloße Rationalitätsfiktionen entlarvt und entzaubert. Einerseits wird damit dem unproduktiven Lagerkampf der Ideologien ein vorläufiges Ende bereitet. Andererseits destabilisiert die Delegitimierung vorgefertigter Handlungsprogramme à la Smith, Keynes oder Hayek die Basis für wirtschaftspolitische Entscheidungen nachhaltig. Sie sorgt für Verunsicherung, Reformstau und eine Politik der kleinen Schritte. Sie erschüttert insbesondere in Krisenzeiten den Glauben an die Möglichkeit rationaler Entscheidungen. Die schließlich selegierte Expertenmeinung, darin sind sich alle einig, kann sich schon im nächsten Moment als falsch herausstellen. Es entsteht ein permanenter Korrekturbedarf: „Wer weiß, was noch alles auf uns zukommt...!", heißt es in jedem zweiten Statement zur aktuellen Wirtschaftslage. Zugleich sind die nationalstaatlichen Regierungen zum Handeln ge- zwungen. Jegliches Zögern wird als Führungsschwäche interpretiert und birgt die Gefahr, für eventuelle Folgeschäden des Abwartens haftbar gemacht zu werden. Und so tasten sich die nationalstaatlichen Regierungen vorwärts. In Deutschland gab es ein erstes Rettungspaket (September 2008), dann ein zweites (Januar 2009) und demnächst vermutlich ein drittes und viertes. Doch je weiter sich die Krise ausbreitet und auf den Arbeitsmarkt übergreift, desto schwieriger wird die Frage, mit welchen politischen Mitteln die Kettenreaktion noch gestoppt werden kann.

Im folgenden Abschnitt richten wir unser Augenmerk verstärkt auf die zeitliche Komponente des Entscheidens. Die geschilderte Problematik im Hinblick auf soziale und sachliche Komplexität ließe sich vermutlich abmildern, wenn staatliche Politik ersatzweise auf die Metaressource Zeit zurückgreifen könnte.

\section{Polit \\ Politik und Beschleunigung}

Uwe Schimank (2005) stellt in seinem Buch zur Entscheidungsgesellschaft die Vermutung an, dass sich mit einem unendlich großen Zeitbudget alle Entscheidungsprobleme, die durch Komplexitätsdruck entstehen, überwinden ließen. Politische Entscheidungsträger könnten sich dann mit den exotischen Gestalten und Produkten vertraut machen, die sich mittlerweile auf den entfesselten Märkten versammelt haben: Hedgefonds, Leerverkäufe, Dark Pools, Credit Recovery Swaps, etc. Die „Giftpapiere" in den Bankbilanzen könnten einer genauen Untersuchung unterzogen werden, um die dort noch schlummernden Risiken realistisch einschätzen zu können. Und schließlich würden milliardenschwere Rettungspakte erst dann verabschiedet, wenn eine sorgfältige Analyse der Krise und ihrer Ursachen vorgenommen wurde. Kurzum: Ohne Zeitdruck wäre es möglich, empirische Evidenzen zu sammeln und somit eine stabile(re) Entscheidungsgrundlage zu konstruieren.

Die Realität sieht indes anders aus. Im Fall der staatlichen Politik stellt sich nämlich das Problem, dass den Entscheidungsträgern selbst das vorhandene, zutiefst irdische Zeitbudget unter den Händen zu zerrinnen scheint. Während sich die weltgesellschaftliche Umwelt dynamisiert, scheint der demokratische Willensbildungsprozess seine quasi-natürliche Geschwindigkeitsgrenze längst erreicht zu haben. Die institutionellen Eigenzeiten des parlamentarischen Prozesses sind in den letzten Jahrzehnten nahezu unverändert geblieben (Riescher 1994). Die Politik wird dabei vom Schrittmacher gesellschaftlicher Entwicklungen zum Bremsklotz der Moderne (Rosa 2005b, S. 391ff.). Demokratische Selbststeuerung ist ohne Zweifel ein zeitaufwendiges Verfahren; es bedarf mühsamer Interessenartikulation, -aggregation und zeitraubender Konsensfindung - und genau diese Zeitressourcen scheinen angesichts des hohen Tempos sozialer und technischer Wandlungsprozesse nicht länger vorhanden zu sein. Tatsächlich, so lässt sich zeigen, nimmt die Zeit, die den Parlamentariern pro Entscheidung zur Verfügung steht, kontinuierlich ab, während der Bedarf, um sachliche und soziale Rationalitätsniveaus zu erhalten, sogar steigt (dazu ausführlich Rosa 2005a). Hierfür lassen sich mindestens drei Gründe angeben: Weil moderne Gesellschaften im Zuge ihrer Pluralisierung und Reflexivierung immer weniger auf Traditionen, Routinen und Konventionen setzen können, steigen - erstens - Zahl und Umfang der regelungsbedürftigen Sozialmaterien. Für immer mehr Felder des Zusammenlebens müssen kollektiv verbindliche Entscheidungen gefällt werden. Zugleich dauert es aber länger, einen Konsens zu erzielen oder auch nur die Interessen zu artikulieren und zu organisieren. Weil gleichzeitig, wie wir bereits gezeigt haben, die (globalen, ökonomischen, kulturellen und ökologischen) Hintergrundbedingungen des Handelns instabiler werden und die sachliche, räumliche und zeitliche Reichweite der Interdependenzketten ansteigt (Beck 2007), benötigen politische Entscheider - zweitens - de facto sogar mehr Zeit, die Konsequenzen und Effekte der Entscheidungsalternativen hinreichend zu durchdenken. Weil sich dabei aber andere gesellschaftliche Felder, besonders Wissenschaft, Technik und Ökonomie, seit den digitalen und politischen Revolutionen um 1989/90 noch einmal deutlich beschleunigt haben, steigt - drittens - auch das Tempo, mit dem soziale Felder re-reguliert werden müssen, in dem also bereits

Eben darin identifiziert bekanntlich Ulrich Beck ein zentrales Signum der "zweiten“ oder "reflexiven“ Moderne (Beck et al. 1996). 
entschiedene Materien wiedervorgelegt werden und neuer Regelungen bedürfen. Die Folge dieser Entwicklungen ist eine massive Desynchronisation zwischen dem Tempo politischer Entscheidungen und dem Tempo des sozialen Wandels. Politik kommt daher chronisch zu spät.

Empirische Studien für den Deutschen Bundestag zeigen, dass die Abgeordneten bei weitgehend konstant bleibender Sitzungszeit mehr Gesetze in das Parlament einbringen und verkünden als jemals zuvor. Sie dürfen sich nicht nur immer häufiger entscheiden, sondern sie müssen es offensichtlich auch. $90 \%$ von ihnen geben gemäß einer Erhebung des Jenaer Sonderforschungsbereichs (SFB 580) aus dem Jahr 2007 an, dass ihnen nicht genügend Zeit zum Nachdenken über anstehende Probleme bleibt. Die Politik wird somit in die Defensive gedrängt. Entscheidungen stehen unter einem immer höheren Zeitdruck, der mit den zeitintensiven Willensbildungsprozessen in den Parlamenten nicht vereinbar ist.

\subsection{DAS DESYNCHRONISATIONS- PROBLEM}

Unseren Beobachtungen zufolge verschärfen sich gegenwärtig die bereits vorhandenen Rhythmusunterschiede und verursachen eine wachsende temporale Desynchronisation zwischen Politik und gesellschaftlicher Umwelt. Diese Tendenz wird in der aktuellen Weltfinanzkrise beinahe idealtypisch erkennbar. Temporale Brüche entstehen hier insbesondere im Verhältnis zwischen Politik, Medien und Wirtschaft: Operationsgeschwindigkeit und Reaktionszeit der drei Systeme differieren erheblich. So ist etwa das Entscheidungstempo eines Parlaments im Vergleich zu einer Börse oder einer Tageszeitung äußerst gering. Die Redaktion einer Tageszeitung entscheidet Tag für Tag aufs Neue, welche Aspekte des Weltgeschehens relevant sind und wie diese zu bewerten sind. Noch schneller operiert die Börse: Sie reagiert auf kleinste Veränderungen der wirtschaftlichen oder politischen Umwelt und drückt ihre „Entscheidung" in Form von veränderten Aktienkursen aus. Im Gegensatz dazu zeigt sich der politische Diskurs zwar ebenfalls sensibel für Veränderungen seines Umfeldes, allerdings findet eine entscheidungsförmige Reaktion im engeren Sinne erst mit großer Zeitverzögerung statt. Die durchschnittliche Dauer zwischen Einbringung und Verabschiedung eines Gesetzesentwurfs beträgt für den Deutschen Bundestag im Fall von zustimmungspflichtigen Gesetzen 225 Tage (Schindler 2000, S. 2414). Diese eklatante Desynchronisation zwischen Politik und Wirtschaft/Medien potenziert die Probleme in der aktuellen Finanzkrise beträchtlich. Bevor das erste Konjunkturpaket des Bundes überhaupt in Kraft getreten war, wurde in den Medien bereits ein zweites Paket gefordert, das die Schwachstellen des ersten beseitigen sollte. Gerade in einer Phase, in der offenbar jeden Tag ein anderes Großunternehmen kurz vor der Insolvenz steht, erweist sich demokratische Politik als zu langsam.

\subsection{LEHMAN BROTHERS - EIN BEISPIEL TEMPORALER DESYNCHRONISATION}

Als sich im September 2008 die amerikanische Regierung dazu entscheidet, den $\mathrm{Zu}-$ sammenbruch der Investmentbank Lehman Brothers nicht zu verhindern, bricht das weltweite Finanzsystem wie ein Kartenhaus in sich zusammen. War das eine durchdachte, kluge, eine weitsichtige, eine notwendige, eine rationale Entscheidung der US-Regierung? Vielleicht sollte man die Frage herumdrehen: Inwiefern war unter den gegebenen Umständen überhaupt eine kluge Entscheidung möglich? Vor dem Hintergrund der von uns entfalteten Argumentation ist zu prüfen, ob die notwendigen Ressourcen vorhanden waren, um eine rationale Entscheidung im Sinne der oben identifizierten Kriterien zu treffen. Die USRegierung räumt mittlerweile ein, dass sie im Fall Lehman eine Fehlentscheidung getroffen hat. Die Frage nach dem erreichten Grad an substanzieller (Ergebnis-)Rationalität ist damit aus Sicht der Verantwortlichen bereits (negativ) beantwortet. Inwiefern ist dem damaligen Beschluss aber ein rationaler Entscheidungsprozess vorausgegangen? Im Fall der Investmentbank Lehman Brothers Holdings Inc. hatte die USRegierung exakt fünf Tage Zeit, um auf die existenzielle Krise der Bank zu reagieren. Am 10.9.2008 verkündete der Vorstand von Lehman Brothers, dass die lebensnotwendige Kapitalerhöhung gescheitert sei: Der potenzielle Finanzinvestor (Korean Development Bank) hatte von einer Beteiligung Abstand genommen. Zugleich wurde lanciert, dass für das dritte Quartal 2008 ein Verlust von 3,9 Milliarden Dollar zu erwarten sei. Die viertgrößte Investmentbank der
USA, mit ihren damals 28.000 Angestellten, war von einem Tag auf den anderen illiquide. Daraufhin kam der US-amerikanische Staat als letzter Retter der Großbank ins Gespräch. Die politische Entscheidung über Einmischung oder Nichteinmischung konnte in diesem Fall allerdings schwerlich im Rahmen der dafür vorgesehenen, institutionellen Bahnen getroffen werden: Das hätte schlicht und ergreifend zu lange gedauert. Stattdessen trafen sich einige Repräsentanten der US-Regierung (unter dem Vorsitz des Finanzministers Paulson) mit den Vertretern von Lehman und den Chefs der anderen US-amerikanischen Großbanken zu einem Sondergipfel. Nach einem erfolglosen Verhandlungsmarathon wurde am 15. September 2008 das Insolvenzverfahren im Fall Lehman eingeleitet. Anders als ein paar Tage zuvor, als die angeschlagenen Hypothekenbanken Fannie Mae und Freddie Mac quasi in letzter Minute vor dem Konkurs bewahrt wurden, entschied sich die US-Regierung gegen Rettung und Staatsbeteiligung. Das erforderliche Informations- und Zeitbudget, um die Entscheidungsgrundlage zu stabilisieren, war schlichtweg nicht vorhanden. Die Ungleichzeitigkeit zwischen politischer Deliberations- und ökonomischer Zirkulationssphäre ist in diesem Fall eklatant. Zum Zeitpunkt des Beschlusses kannte niemand die genaue Position von Lehman im Netz der Weltwirtschaft, geschweige denn Zahl, Art und Umfang ihrer Geschäftsbeziehungen. Selbst aus heutiger Perspektive und der zeitlichen Distanz von mehreren Monaten ist weitgehend ungeklärt, in welchem Ausmaß weitere Banken und Unternehmen von der geplatzten Kreditblase betroffen sind. Das erreichte Niveau an Prozessrationalität der damaligen Regierungsentscheidung muss somit als äußerst niedrig veranschlagt werden. Die Voraussetzungen für einen klugen Entschluss waren nicht gegeben, das Ergebnis ist bekannt.

\subsection{VERGLEICHBARE ENTSCHEIDUNGSBEDINGUNGEN: DAS KONJUNKTURPAKET II}

Unter ähnlich schwierigen Vorzeichen operiert derzeit die politische Klasse Deutschlands. Mit Rettungsschirmen und Konjunkturprogrammen kämpft sie um Sparkonten, Arbeitsplätze, die Autobranche und die Stabilisierung des heimisch-globalen Finanzmarktes. Die temporalen Paradoxien lassen sich exemplarisch anhand 
des Konjunkturpaktes II skizzieren. Trotz des Superwahljahres 2009 haben sich die Regierungsparteien der Großen Koalition in erstaunlich kurzer Zeit auf ein Paket gigantischen Ausmaßes (ca. 50 Mrd. €) geeinigt. Unter tatkräftiger Mitwirkung der Oppositionsparteien wurde die parlamentarische Geschäftsordnung zum Teil außer Kraft gesetzt. Auf diese Weise konnte das Paket dem Bundestag bereits Ende Januar 2009 zur Diskussion vorgelegt werden. Das Bemühen der Beteiligten um eine Beschleunigung des demokratischen Entscheidungsprozesses ist also kaum zu übersehen. Und doch beklagten fast alle Experten, dass das Konjunkturpaket viel zu spät komme. An dieser Stelle macht sich, trotz aller Eile, vor allem die Dauer zwischen Einbringung, Verabschiedung und Umsetzung eines Gesetzesvorhabens negativ bemerkbar. Das Paket wurde am 12.1.2009 vom Koalitionsausschuss gebilligt. Am 27.1. stimmte das Bundeskabinett zu, am 13.2. der Bundestag und am 20.2. der Bundesrat. Am 1.7.2009 trat das Paket schließlich in Kraft. Ein Teil des Geldes geht an die Landesregierungen, die wiederum entscheiden müssen, wofür sie das Geld ausgeben wollen. Die geplanten Maßnahmen, etwa zum Ausbau der Infrastruktur, müssen dann erst noch öffentlich ausgeschrieben werden. Vermutlich wird es daher mindestens bis Ende 2009 dauern, also fast ein Jahr, bis das Paket die schwer beschädigte Wirtschaft ankurbeln kann. In der Zwischenzeit kann allenfalls auf die psychologische Wirkung des Maßnahmenpakets gehofft werden. Bis dahin wird sich die Politik wohl noch häufiger den Vorwurf gefallen lassen müssen, dass ihre Aktionen zu spät greifen. Wirtschaftliche Entwicklung und massenmediale Vermittlung diktieren das Tempo, an dem Politik täglich gemessen wird.

Was hier exemplarisch skizziert wurde, mag in seiner Radikalität spezifisch für die aktuelle Finanzkrise sein, doch ist es in seiner Tendenz paradigmatisch für das politische Handeln im Zeitalter der Globalisierung: Die hohe Komplexität der Entscheidungsprobleme sowie der immense Zeitdruck von außen können durch nationalstaatliche Politik kaum noch verarbeitet werden. Die (heterogenen) Forderungen der Bürger, Gewerkschaften und Konzerne treffen auf eine politische Klasse, der es nicht so sehr an Entschlossenheit oder Verantwortungsgefühl mangelt, sondern vielmehr an den existenziellen Ressourcen für nachhaltig gute Beschlüsse. Wie gehen die Entscheidungsträger mit der hier skizzierten Situation um?

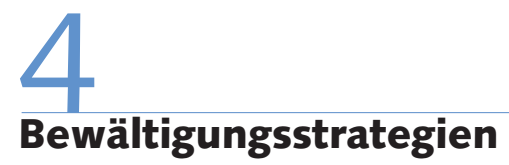

Politische Entscheidungsträger haben in der Vergangenheit verschiedene Wege gefunden, mit den Entscheidungszumutungen der Spätmoderne umzugehen. Auf einige der Möglichkeiten haben wir bereits hingewiesen. So lässt sich der Teilrückzug des Staates aus den Finanzmärkten dadurch erklären, dass die politischen Repräsentanten das Risiko politischer Fehlentscheidungen in diesem Feld als zu groß wahrnahmen. ${ }^{6}$ Darüber hinaus haben wir auf das Bemühen um eine Beschleunigung des parlamentarischen Verfahrens hingewiesen. Dieser Versuch kristallisiert sich besonders deutlich heraus in der parteiübergreifend gebilligten Umgehung der parlamentarischen Geschäftsordnung, als es um die Konjunkturpakete I und II ging. ${ }^{7}$ Diese und andere Strategien zeigen, dass eines offenbar unter allen Umständen vermieden werden muss: Eine Erschütterung des Glaubens an die Rationalität der Politik. Weltfinanzkrise oder nicht, nationalstaatliche Politik kann diesem Imperativ letztlich nicht entkommen. Undenkbar, dass eine Bundeskanzlerin oder ein Bundeskanzler zur traditionellen Neujahrsansprache vor die Mikrofone der Öffentlichkeit tritt und zugibt, sich unter den gegebenen Umständen nicht in der Lage zu sehen, rationale Entscheidungen zu treffen. Wer an einer Fortsetzung seiner politischen Karriere interessiert ist, darf sich keinerlei Verunsicherung anmerken lassen. Politikerinnen und Politiker müssen vielmehr stets gute Gründe für ihre Beschlüsse geltend machen - insbesondere wenn es sich um milliardenschwere Rettungsmaßnahmen handelt. In diesem Sinne sind politische Repräsentanten gerade in der Krise zur aktiven Reproduktion der Rationalitätsfiktion gezwungen. Sie müssen den Anschein erwecken, dass sie genau wissen, was sie tun und welche Konsequenzen das haben wird. Inoffiziell wird jedoch außerhalb demokratischer Institutionen um die Aufrechterhaltung des vorhandenen "Satisficing“Niveaus gerungen.

Zum Abschluss dieses Beitrags wollen wir auf einen Bewältigungsmechanismus hinweisen, der sich auch und gerade in der aktuellen Krise als besonders wirkmächtig erwiesen hat: Die Konstruktion und Pflege formeller sowie informeller Netzwerke.

Kontaktnetzwerke bieten im Vergleich zu den offiziellen Prozeduren und Institutionen einige Vorteile: Sie ermöglichen den schnellen Austausch von Informationen, die direkte Kommunikation zwischen Wirtschafts- und Politikexperten sowie gegenseitige Hilfestellungen und Koppelgeschäfte fernab des parlamentarischen Geschehens. Netze transzendieren Grenzen: Sie verknüpfen Akteure unterschiedlichster System- und Ordnungsebenen. Entscheidungsrelevante Informationen sind über die Netze daher viel schneller verfügbar: Merkel musste nicht erst auf die nächste Bilanz der Deutschen Bank warten, um zu wissen, inwieweit diese von der Krise betroffen ist. Stattdessen bekam sie die Informationen lange vor ihrer Veröffentlichung von Ackermann persönlich. So konnte innerhalb kürzester Zeit abgeschätzt werden, wie umfangreich der Bankenrettungsschirm tatsächlich ausfallen muss. Die direkte Kommunikation an der Schnittstelle zwischen Politik und Wirtschaft ermöglicht zudem eine effizientere Koordination der Einzelmaßnahmen. Ohne die enge Verbindung zwischen Automobilbranche und deutscher Regierung wäre die schnelle Einigung beim Thema Kurzarbeitergeld sicherlich nicht möglich geworden. Das Entgegenkommen der Politik wurde (vorerst) belohnt: Anstelle von betriebsbedingten Kündigungen schickten die Automobilkonzerne ihre Mitarbeiter Anfang des Jahres, wie abgesprochen, in die Kurzarbeit. Der hohe Verflechtungsgrad zwischen Politik und Wirtschaft garantiert somit ein Vertrauensverhältnis zwischen den beteiligten Akteuren, das offenbar auch in Krisenzeiten paretooptimale (oder wenigstens „paretogute“) Lösungen ermöglicht.

Als prägnanter Beleg für den Bedeutungszuwachs der Exekutivnetze erweist sich der im Oktober 2008 gegründete Sonderfond Finanzmarktstabilisierung (SoFFin).

6 Es hat sich freilich ex post überdeutlich erwiesen, dass die Zurückhaltung des Staates sogar noch größere Risiken barg: Selbst diese Rückzugsstrategie offenbart sich daher als eine letztlich irrationale Entscheidung!

7 Tatsächlich lassen sich in nahezu allen Ländern der Erde solche parlamentarischen und außerparlamentarischen Beschleunigungsinitiativen beobachten (Rosa 2005a, S. 403f.; Scheuerman 2004). 
Zugleich offenbart sich an diesem Exempel das Kernproblem netzbasierter Entscheidungsprozesse. Als Schnittstelle zwischen Politik und Wirtschaft befindet der SoFFin über milliardenschwere Staatshilfen und Bürgschaften für angeschlagene Unternehmen und Banken. Personell ist die Institution lediglich mit einer Handvoll Experten (zurzeit 23 Personen) aus Wirtschaft und Politik bestückt. Entsprechende Hilfspakete werden im direkten Kontakt mit den unternehmerischen Abgesandten ausgehandelt. Finanzhilfe wird hier vergleichsweise schnell und unkompliziert gewährt. Im Fall der Commerzbank wurde Ende 2008/Anfang 2009 innerhalb von vier Wochen über Hilfsleistungen im Wert von 18,2 Mrd. € entschieden. Netzförmige Gebilde wie der SoFFin eignen sich offenbar ausgezeichnet zur Beschleunigung politischer Entscheidungsprozesse. Diese Schnelligkeit hat allerdings ihren Preis: Der SoFFin unterliegt im Rahmen seiner Tätigkeit nämlich nicht der parlamentarischen Kontrolle des Bundeshaushaltsgesetzes. Ein Mitspracherecht des Bundestages bei der Vergabe der Gelder wurde vielmehr unter anderem mit der Begründung zurückgewiesen, dass im Rahmen der Finanzkrise schnelle Entscheidungen gefragt seien. Die Legislative wird in unsicheren Zeiten also zunehmend als Hindernis wahrgenommen. ${ }^{8}$ Ihre verfassungsmäßig fundierte Logik, die kritische Begleitung des Regierungshandelns, sperrt sich gegen die schnelle Reduktion von Komplexität. Im Gegensatz zum SoFFin und anderen hybriden Netzwerkstrukturen wird die Komplexität von Entscheidungsproblemen im Parlament nicht vorschnell reduziert, sondern im Rahmen von zeitaufwendigen Debatten überhaupt erst in ihrer Gänze entfaltet. Das Parlament ist keine Hilfe, wenn es um die schnelle Kons- truktion einer Entscheidungsgrundlage geht. Die intersubjektiven Verständigungsprozesse innerhalb der demokratischen Infrastrukturen zielen auf Rationalität, nicht auf Beschleunigung.

Die Steigerung des Beschleunigungsund Komplexitätsdrucks lässt die stärkere Verschränkung und Vernetzung von Politik und Wirtschaft in Krisenzeiten geradezu als Sachzwang erscheinen. Politik spielt sich daher gegenwärtig vor allem auf eilig einberufenen Job-, Mittelstands-, Gewerkschafts-, Arbeitgeber-, Banken- und Autogipfeln ab. Auf diese Weise gelingt dem Staat zwar eine Tempoverschärfung, das Rationalitätsniveau der getroffenen Entscheidungen bleibt indes bestenfalls konstant (niedrig). Fluchtpunkt der Vernetzung ist nicht die Rationalität der einzelnen Entscheidungen, sondern die Schnelligkeit und Durchsetzbarkeit der entsprechenden Politikentwürfe. Die Appelle zur ergebnisoffenen diskursiven Auseinandersetzung über Politikziele und Instrumente bleiben innerhalb der Netzwerkstrukturen notwendig ungehört. Politische Rationalität wird zur Fiktion.

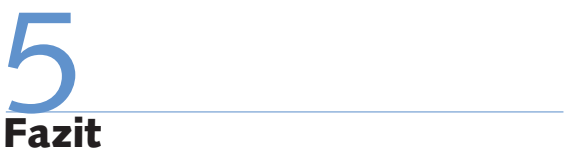

Wie wir gezeigt haben, wird Politik im Zuge der gesellschaftlichen Veränderungsprozesse auf merkwürdige Weise willkürlich. Es ist für Beobachter und Teilnehmer nicht mehr erkennbar, was "richtige“ Entscheidungen sein könnten. Nach wie vor ist es möglich, irgendwelche Gründe für eine Selektion anzugeben. Aber wie sieht es mit „richtig“ guten Gründen aus? Die Voraussetzungen für rationale Entscheidungspro- zesse, so unser Kernargument, werden zunehmend untergraben. Diese Beobachtung behält auch dann ihre Gültigkeit, wenn Rationalität in der verkürzten Lesart der Rational-Choice-Theorien als „egoistische Nutzenmaximierung" definiert wird: Langfristige politische Planung wird in der Gegenwart, ganz unabhängig von den verfolgten Präferenzen, geradezu dysfunktional und muss durch flexibel-kurzfristiges Anpassungsverhalten ersetzt werden. Eine Politik des „muddling through" (Lindblom 1959) ist nicht nur in Krisenzeiten der sichtbare Effekt. Durch das Instrument der Vernetzung gelingt ein letzter verzweifelter Beschleunigungsschub, der jedoch allenfalls den Rückstand konstant hält. Im Schatten der Netzwerke wird indes der demokratische Willensbildungsprozess unterhöhlt - und damit die Hoffnung auf politische Rationalität, da eine demokratischdeliberative, kollektive Präferenzbildung ausbleibt. Legt man die hier definierten Rationalitätskriterien zugrunde, dann scheint es im Licht der von uns geprüften Argumente unabweisbar, dass die Rationalität politischer Entscheidungen unaufhaltsam sinkt. Paradoxerweise sind die politischen Repräsentanten just in einem Klima der Unentscheidbarkeit zum Einsatz von vielen hundert Milliarden Euro Steuergeld aufgerufen. Unter den gegebenen Umständen kann man dem Staat bei seinem „Comeback“ auf dem internationalen Finanzparkett im Grunde nur viel Glück wünschen.

8 Eine ausführliche Begründung für das Argument, dass der Beschleunigungsdruck der Spätmoderne das Kräfteverhältnis zwischen einer bürgergesellschaftlich verankerten Legislative und der Exekutive progressiv zugunsten der Letzteren verschiebt, hat Scheuerman (2009) geliefert. 


\section{LITERATUR}

Beck, U. (2007): Weltrisikogesellschaft: Auf der Suche nach der verlorenen Sicherheit, Frankfurt/M.

Beck, U./Giddens, A./Lash, S. (1996): Reflexive Modernisierung. Eine Kontroverse, Frankfurt/M

Brost, M./Schieritz, M./Storn, A. (2008): Mit vollen Händen, in: Die Zeit 50 vom 4.12. Online abrufbar unter: http://www.zeit.de/2008/50/ Staat-und-Banken

Habermas, J. (1985): Die Krise des Wohlfahrtsstaates und die Erschöpfung utopischer Energien, in: Ders (Hrsg.): Die neue Unübersichtlichkeit, Frankfurt/M, S. 141-163

Lessenich, S. (2008): Die Neuerfindung des Sozialen. Der Sozialstaat im flexiblen Kapitalismus, Bielefeld

Lindblom, C. E. (1959): The Science of "Muddling Through“, in: Etzioni, A. (Hrsg.): Readings on Modern Organizations, Englewood Cliffs, S. 154-166 Luhmann, N. (1994): Die Wirtschaft der Gesellschaft, Frankfurt/M.

Mayntz, R./Scharpf, F. W. (2005): Politische Steuerung - Heute?, in: Zeitschrift für Soziologie 3, S. 236-243

Patzelt, W. (1996): Deutschlands Abgeordnete. Profil eines Berufsstandes, der weit besser ist als sein Ruf, in: Zeitschrift für Parlamentsfragen 3, S. 462-502

Riescher, G. (1994): Zeit und Politik. Zur institutionellen Bedeutung von Zeitstrukturen in parlamentarischen und präsidentiellen Regierungssystemen, Baden-Baden

Rosa, H. (2005a): The Speed of Global Flows and the Pace of Democratic Politics, in: New Political Science 4, S. 445-459
Rosa, H. (2005b): Beschleunigung. Die Veränderung der Zeitstrukturen in der Moderne, Frankfurt/M

Scheuerman, W. (2004): Liberal Democracy and the Social Acceleration of Time, Baltimore

Scheuerman, W. (2009): Citizenship and Speed, in: Rosa, H./Scheuerman, W. (Hrsg.): High Speed Society. Social Acceleration, Power and Modernity, S. 287-306

Schimank, U. (2005): Die Entscheidungsgesellschaft. Komplexität und Rationalität der Moderne, Wiesbaden

Schimank, U. (2006): Rationalitätsfiktionen in der Entscheidungsgesellschaft, in: Tänzler, D./Knoblauch, H./Soeffner, H.-G. (Hrsg.): Zur Kritik der Wissensgesellschaft. Erfahrung - Wissen - Imagination. Schriften zur Wissenssoziologie, Konstanz, S. 57-81

Schindler, P. (2000): Datenhandbuch zur Geschichte des Deutschen Bundestages 1949-1999, Gesamtausgabe in drei Bänden, Bd. 1, BadenBaden

Simon, H. A. (1946): Administrative Behaviour. A Study of DecisionMaking Processes in Administrative Organization, New York Simon, H. A. (1993): Homo rationalis. Die Vernunft im menschlichen Leben, Frankfurt/M.

Soeffner, H.-G. (2000): Gesellschaft ohne Baldachin, Weilerswist Taylor, C. (1996): Quellen des Selbst. Die Entstehung der neuzeitlichen Identität, Frankfurt/M. 